\title{
La importancia de rehabilitar tepetate con Lupinus campestris Cham. \& Schltdl.
}

The importance of tepetate rehabilitation with Lupinus campestris Cham. \& Schltdl.

Munive-Martínez, C. ${ }^{1}$, Vázquez-Cuecuecha, O. ${ }^{1}$, Zamora-Campos, E. ${ }^{1}$, López-López, A. ${ }^{2}$, Calvario-Rivera, C. I. ${ }^{2}$ García-Gallegos, $\mathrm{E}^{1 \otimes}$.

${ }^{1}$ Maestría en Ciencias en Sistemas del Ambiente.

${ }^{2}$ Cátedra CONACyT. Centro de Investigación en Genética y Ambiente. Universidad Autónoma de Tlaxcala.Av. Universidad No. 1, Col. La Loma Xicohténcatl, Tlaxcala. CP. 90000. Tel/Fax. 012484815500.

${ }^{\bowtie}$ Autor para correspondencia: gallegoseg@ hotmail.com

Recibido: 13/07/2018

Aceptado: 12/09/2018

\section{RESUMEN}

El tepetate es un horizonte endurecido con baja retención de humedad y fertilidad, restrictivas para el desarrollo de especies vegetales; sin embargo, puede mejorar esta condición a través de prácticas de rehabilitación, como lo es el empleo de leguminosas. Se determinó la potencialidad de diferentes poblaciones de Lupinus campestris como alternativa para rehabilitar y mejorar la calidad productiva de los tepetates de la hacienda San Antonio Tepetzala, localizada en el municipio de Atlangatepec, Tlaxcala. Se estableció un diseño experimental en bloques completamente al azar, los tratamientos fueron nueve poblaciones de L. campestris procedentes del estado de Puebla. A los 12 meses se llevó a cabo el muestreo de tepetate de la zona rizosférica de las leguminosas, para evaluar el $\mathrm{pH}$, conductividad eléctrica, materia orgánica, capacidad de intercambio catiónico, $\mathrm{N}$ total, $\mathrm{P}, \mathrm{K}, \mathrm{Ca}$ y $\mathrm{Mg}$. Los resultados mostraron una diferencia significativa $(\mathrm{p}<0.05)$ después del establecimiento de la planta,pero no entre poblaciones. Si bien los valores de cada una de las variables determinadas con base a la normatividad mexicana fueron bajos,es notoria la mejora a un año de establecer diferentes poblaciones de L. campestris en el tepetate. Por lo que se concluye que L. campestris puede mejorar las características del tepetate, lo que la convierte en una excelente candidata para la rehabilitación de zonas degradadas.

Palabras clave: Propiedades edáficas, leguminosas, roturación, horizontes endurecidos.

\begin{abstract}
Tepetate is a hard horizon with low humidity and fertility retention, restrictive to plant species development; although this condition can be improved through rehabilitation practices, for example with the use of legumes. The potential of different Lupinus campestris populations was determined as an alternative to rehabilitate and improve the productive quality of tepetate of the Hacienda San Antonio Tepetzala, located in Atlangatepec municipality, Tlaxcala. An experimental design was established in completely random blocks, the treatments were nine L. campestris populations from
\end{abstract}


Puebla State. After 12 months, lupines growth on tepetate, $\mathrm{pH}$, electric conductivity, organic matter, cation exchange capacity, total $\mathrm{N}, \mathrm{P}, \mathrm{K}, \mathrm{Ca}$ and $\mathrm{Mg}$ were evaluated from plant rhizospheric zone. The results showed significant difference $(\mathrm{p}<0.05)$ after plant establishment, but not among populations. Although data from each variabledetermined with the Mexican norms were low, the improvement is remarkable one year after of diverse L. campestris populations establishment on tepetate, which makes it an excellent candidate for degraded areas rehabilitation.

Keywords: Edaphic properties, legumes, roturation, hardened horizons.

\section{INTRODUCCIÓN}

El proceso de degradación del suelo avanza con una mayor rapidez que las medidas tomadas para su prevención y control; la carencia de protección por cubierta vegetal da como consecuencia el afloramiento del tepetate en algunas zonas del centro de la República Mexicana. Gama-Castro et al., (2007) señalan que el término tepetate se refiere a un horizonte endurecido, ya sea compactado o cementado que se puede encontrar bajo los suelos o bien aflorando en la superficie. Presenta características físicas, mecánicas y químicas restrictivas para el desarrollo de la vegetación; tales como, alta densidad aparente, baja conductividad hidráulica, poca fertilidad y una importante retención de humedad. Es por ello que para iniciar la rehabilitación de este sustrato se debe practicar una roturación, posteriormente nivelar, y realizar bordes para el establecimiento de plantas o simplemente aplicar fertilizantes para favorecer su capacidad nutrimental (Báez et al., 2008; Pajares-Moreno et al., 2010).

Diversas prácticas han sido reportadas para mejorar las condiciones edáficas del tepetate; como son el empleo de leguminosas y hongos endomicorrízicos arbusculares (GarcíaGallegos et al., 2009); así como la rotación de cultivos (Flores et al., 2004). En este sentido, se ha reportado que especies del género Lupinus (Leguminosae) presentan la capacidad de solubilizar fósforo, gracias a su sistema radical extensivo y a su potencial de supervivencia y desarrollo en suelos con escasez de agua y deficientes en nutrimentos (Vielma, 1999). $L$. campestris es una especie que crece en suelos degradados del centro de México y posee potencial para mejorar la fertilidad del suelo, al fijar $\mathrm{N}_{2}$ atmosférico y solubilizar P; por lo cual, puede ser considerada en la restauración ecológica de suelos degradados (Vance, 2001; Gutiérrez et al., 2010). Por lo anterior, el objetivo de la presente investigación consistió en evaluar las propiedades edáficas y el contenido nutrimental de un área con tepetate, empleando diferentes poblaciones de Lupinus campestris para contribuir a su rehabilitación y mejorar su calidad productiva.

\section{MATERIALES Y MÉTODOS}

El área de estudio es el tepetate de la Hacienda San Antonio Tepetzala, en el municipio de Atlangatepec, estado de Tlaxcala; esta se localiza en las coordenadas 19³2'08.41' $\mathrm{N}$, 98 06'41.73" O a una altitud de $2628 \mathrm{msnm}$. La precipitación media anual del municipio es de $680 \mathrm{~mm}$ y la temperatura promedio anual de 12 a $14^{\circ} \mathrm{C}$ (INEGI, 2009).

Las poblaciones de L. campestris procedieron del estado de Puebla (Tabla 1). 200 semillas de cada población fueron desinfectadas con $\mathrm{H}_{2} \mathrm{O}_{2}$ al $10 \%$ por 10 min y se escarificaron con una lija para madera (Vázquez, 2017), posteriormente fueron sembradas de forma directa en tubetes de plástico negro en un 
sustrato compuesto por corteza de pino, aserrín y agrolita en una proporción 2:1:1 (p/p/p). Se mantuvieron las plántulas a humedad constante por 60 días, bajo condiciones de invernadero.

Tabla 1. Poblaciones de semillas de L. campestris del estado de Puebla.

\begin{tabular}{llccc}
\hline \multicolumn{1}{c}{ Población } & Latitud & Longitud & Altura (msnm) \\
\hline 1 & Poxcoatzingo(POX) & $19^{\circ} 57^{\prime} 13.0^{\prime \prime}$ & $98^{\circ} 00^{\prime} 33.5^{\prime}$ & 2481 \\
2 & Laguna de Atexca (LAT) & $19^{\circ} 57^{\prime} 09.0^{\prime \prime}$ & $98^{\circ} 01^{\prime} 25.0^{\prime \prime}$ & 2503 \\
3 & Zoapan Sitio A (ZOA) & $19^{\circ} 04^{\prime} 32.3^{\prime \prime}$ & $97^{\circ} 20^{\prime} 18.1^{\prime \prime}$ & 3200 \\
4 & Zoapan Sitio B (ZOB) & $19^{\circ} 04^{\prime} 34.8^{\prime \prime}$ & $97^{\circ} 22^{\prime} 04.2^{\prime \prime}$ & 3112 \\
5 & Laguna Seca (LSC) & $18^{\circ} 63^{\prime} 30.5^{\prime \prime}$ & $97^{\circ} 19^{\prime} 0.06^{\prime}$ & 2669 \\
6 San Isidro (SIS) & $18^{\circ} 54^{\prime} 33.6^{\prime \prime}$ & $97^{\circ} 18^{\prime} 26.4^{\prime \prime}$ & 2794 \\
7 & Barranca Honda (BAH) & $19^{\circ} 01^{\prime} 46.0^{\prime \prime}$ & $97^{\circ} 22^{\prime} 43.1^{\prime \prime}$ & 2903 \\
8 & Tlalmotolo (TLAL) & $19^{\circ} 34^{\prime} 27.4^{\prime \prime}$ & $97^{\circ} 43^{\prime} 38.5^{\prime \prime}$ & 2980 \\
9 & Altzizintla (ALZ) & $18^{\circ} 53^{\prime} 40.0^{\prime \prime}$ & $97^{\circ} 17^{\prime} 57.8^{\prime \prime}$ & 2747 \\
\hline
\end{tabular}

La plantación se realizó en un área donde previamente se establecieron especies del género Opuntia y Pinus en el año 2012. Se usó un diseño experimental de bloques completos al azar, donde las nueve poblaciones de $L$. campestris fueron los tratamientos, cada unidad experimental consistió de seis individuos por poblaciónen una cepa común.

Las muestras de tepetate se recolectaron en el año 2017, 12 meses antes de establecer las plantas de Lupinus y a la cosecha de semilla, en el año 2018 al término del ciclo vegetativo. El muestreo fue de la zona rizosférica de la planta, las muestras se introdujeron en bolsas de plástico y procesaron de acuerdo a lo que establece la NOM-021-SEMARNAT-2000 (DOF, 2002). A cada una se les determinó el $\mathrm{pH}$ en una suspensión de suelo:agua $(1: 2 \mathrm{p} / \mathrm{v}) \mathrm{y}$ materia orgánica (MO) por el método de Walkley-Black; conductividad eléctrica en una suspensión de suelo:agua (1:5 p/v) (ÁlvarezSánchez y Marín-Campos, 2011); la capacidad de intercambio catiónico (CIC) por titulación con $\mathrm{HCl} 0.05 \mathrm{~N}$ (Fuentes, 1971); la concentración de $\mathrm{N}$ total por el método Kjeldahl; los cationes intercambiables (K, Ca y
$\mathrm{Mg}$ ) por la técnica con acetato de amonio y el $\mathrm{P}$ extractable por (Álvarez-Sánchez y MarínCampos, 2011).

Los datos de las diferentes variables evaluadas se sometieron a un análisis descriptivo, de varianza ya una prueba de comparación de medias de Tukey $(p \leq 0.05)$, bajo un modelo de efectos mixtos, para el ANOVA se determinaron los supuestos de normalidad y de homogeneidad de varianza.

\section{RESULTADOS}

En el Tabla 2 se observa que el tepetate previo a establecer las poblaciones de L. campestris fue significativamente diferente $(\mathrm{p} \leq 0.05)$ al tepetate posterior a la cosecha de la planta en cuanto a las variables edáficas y nutrimentales. De acuerdo a la clasificación que señala la NOM-021-SEMARNAT-2000 (DOF, 2002), el $\mathrm{pH}$ fue neutro en el tepetate previo a colocar el experimento al igual que el sustrato con la población ALZ; mientras que, el tepetate con el resto de las poblaciones de L. campestris fue moderadamente alcalino. La CE indica que en las dos condiciones hay cantidades despreciables de sales. Así mismo, el porcentaje 
de materia orgánica fue relativamente bajo, con base en la normatividad mexicana. En cuanto a la CIC, su valor se considera bajo, tanto en el tepetate previo como el tratado con las poblaciones ALZ, BAH, LAT, POX y SIS; en cambio fue medio con las poblaciones LSC, TLAL, ZOA y ZOB. En el caso del contenido nutrimental, el $\mathrm{N}$ total fue bajo en todos los casos de acuerdo a la clasificación que establece la NOM-021 para suelos volcánicos. Con base a la clasificación de Vázquez (1997) las concentraciones que se obtuvieron deK, Ca y $\mathrm{Mg}$ fueron muy pobres.

Tabla 2. Análisis de varianza de algunas propiedades edáficas y contenido nutrimentalen tepetate de la zona rizosférica de nueve poblaciones de L. campestris.

\begin{tabular}{cccccccccc}
\hline Población & $\mathbf{p H}$ & $\begin{array}{c}\text { CE } \\
\mathrm{dS} \mathrm{m} \mathrm{m}^{-1}\end{array}$ & $\begin{array}{c}\text { MO } \\
(\%)\end{array}$ & $\begin{array}{c}\text { CIC } \\
\mathrm{Cmol}(+) \mathrm{kg}^{-} \\
1\end{array}$ & $\begin{array}{c}\text { Ntotal } \\
(\%)\end{array}$ & $\begin{array}{c}\mathbf{P} \\
(\mathrm{mg} \\
\left.\mathrm{kg}^{-1}\right)\end{array}$ & $\begin{array}{c}\mathbf{K} \\
(\mathrm{mg} \\
\left.\mathrm{kg}^{-1}\right)\end{array}$ & $\begin{array}{c}\text { Ca } \\
\left(\mathrm{mg} \mathrm{kg}^{-}\right.\end{array}$ & $\begin{array}{c}\text { Mg } \\
\left(\mathrm{mg}^{-}\right.\end{array}$ \\
\hline NOM-021 & $6.6-8.5$ & $<1$ & $<4.0$ & $5-25$ & $<0.30$ & $<15$ & $<0.40$ & $<500$ & $<15$ \\
\hline ALZ & $7.19 \mathrm{a}$ & $0.07 \mathrm{a}$ & $0.73 \mathrm{a}$ & $14.26 \mathrm{a}$ & $0.03 \mathrm{a}$ & $3.61 \mathrm{a}$ & $3.55 \mathrm{a}$ & $18.93 \mathrm{a}$ & $9.97 \mathrm{a}$ \\
BAH & $7.77 \mathrm{a}$ & $0.07 \mathrm{a}$ & $0.40 \mathrm{a}$ & $11.13 \mathrm{a}$ & $0.03 \mathrm{a}$ & $3.55 \mathrm{a}$ & $3.68 \mathrm{a}$ & $18.57 \mathrm{a}$ & $9.35 \mathrm{a}$ \\
LAT & $7.55 \mathrm{a}$ & $0.08 \mathrm{a}$ & $0.40 \mathrm{a}$ & $14.38 \mathrm{a}$ & $0.03 \mathrm{a}$ & $3.61 \mathrm{a}$ & $3.20 \mathrm{a}$ & $18.93 \mathrm{a}$ & $8.67 \mathrm{a}$ \\
LSC & $7.74 \mathrm{a}$ & $0.06 \mathrm{a}$ & $0.35 \mathrm{a}$ & $15.38 \mathrm{a}$ & $0.03 \mathrm{a}$ & $3.59 \mathrm{a}$ & $3.60 \mathrm{a}$ & $19.42 \mathrm{a}$ & $8.82 \mathrm{a}$ \\
POX & $7.67 \mathrm{a}$ & $0.09 \mathrm{a}$ & $1.01 \mathrm{a}$ & $12.38 \mathrm{a}$ & $0.03 \mathrm{a}$ & $3.57 \mathrm{a}$ & $3.52 \mathrm{a}$ & $18.57 \mathrm{a}$ & $8.43 \mathrm{a}$ \\
SIS & $7.60 \mathrm{a}$ & $0.06 \mathrm{a}$ & $0.45 \mathrm{a}$ & $14.51 \mathrm{a}$ & $0.03 \mathrm{a}$ & $3.56 \mathrm{a}$ & $3.50 \mathrm{a}$ & $18.25 \mathrm{a}$ & $8.65 \mathrm{a}$ \\
TLAL & $7.53 \mathrm{a}$ & $0.06 \mathrm{a}$ & $0.64 \mathrm{a}$ & $16.88 \mathrm{a}$ & $0.03 \mathrm{a}$ & $3.60 \mathrm{a}$ & $3.53 \mathrm{a}$ & $18.42 \mathrm{a}$ & $9.63 \mathrm{a}$ \\
ZOA & $7.74 \mathrm{a}$ & $0.06 \mathrm{a}$ & $0.40 \mathrm{a}$ & $16.75 \mathrm{a}$ & $0.03 \mathrm{a}$ & $3.67 \mathrm{a}$ & $3.48 \mathrm{a}$ & $19.10 \mathrm{a}$ & $8.98 \mathrm{a}$ \\
ZOB & $7.49 \mathrm{a}$ & $0.07 \mathrm{a}$ & $0.78 \mathrm{a}$ & $16.88 \mathrm{a}$ & $0.03 \mathrm{a}$ & $3.64 \mathrm{a}$ & $3.38 \mathrm{a}$ & $19.43 \mathrm{a}$ & $9.02 \mathrm{a}$ \\
\hline \hline *Tepetate & $6.94 \mathrm{~b}$ & $0.04 \mathrm{~b}$ & $0.30 \mathrm{~b}$ & $10.98 \mathrm{~b}$ & $0.02 \mathrm{~b}$ & $2.93 \mathrm{~b}$ & $2.15 \mathrm{~b}$ & $13.63 \mathrm{~b}$ & $7.90 \mathrm{~b}$ \\
2017 & & & & & & & & & \\
\hline \hline
\end{tabular}

Significancia: Bloques $p=0.0001 ;$ años $p=0.0001$. Medias con distinta letra en la misma columna son estadísticamente diferentes $(p \leq 0.05)$. *Tepetate 2017. Tepetate previo a establecer el experimento.

\section{DISCUSIÓN}

Los valores de las propiedades edáficas son de bajo a medio con base a la NOM-021; no obstante, es ligeramente notoria la mejora a un año de establecer diferentes poblaciones de $L$. campestris en el tepetate, propiedad de la Hacienda Tepetzala (Tabla 2). La importancia de rehabilitar a los tepetates a través de la incorporación de especies vegetales es una estrategia útil para aumentar su productividad (García et al., 2008). Las plantas tienen una influencia significativa sobre las características del tepetate, ya que disgregan y agregan el material, además de aportar al sustrato compuestos orgánicos susceptibles de ser usados por la biota o en los procesos de estructuración (Velázquez et al., 2001). La plantación de especies suculentas y/o leñosas en tepetates son un factor clave, ya que otorgan estabilidad física y promueven interacciones biológicas y químicas benéficas (Callo, 2013). En cambio, las leguminosas presentan una elevada producción de biomasa y ocurrencia en 
una amplia gama de condiciones climáticas y edáficas, lo que les confiere un rol significativo en los estudios de recuperación de suelos degradados, debido a que presentan un mejor aprovechamiento de nutrimentos y mayor capacidad de crecimiento en condiciones adversas de suelo; principalmente, cuando están asociadas a hongos micorrízicos arbusculares y bacterias fijadoras de nitrógeno (Dias et al., 1995). El género Lupinus establece simbiosis con el rizobio de crecimiento lento Brady rhizobium, se ha estimado de 102 a $252 \mathrm{~kg} \mathrm{ha}^{-1}$ la cantidad total de nitrógeno fijado por ciclo fenológico, además de tener la capacidad de solubilizar P (Vielma, 1999).

En cuanto a las características químicas del tepetate, éste puede presentar un $\mathrm{pH}$ de neutro a ligeramente alcalino (6.9 a 7.8), de baja conductividad eléctrica $\left(0.09 \mathrm{dSm}^{-1}\right)$, con una capacidad de intercambio catiónico media de $16.7 \mathrm{Cmol}(+) \mathrm{kg}^{-1}$, y respecto a la concentración de nutrimentos, el contenido de nitrógeno puede ir de 0.01 a $0.07 \%$, de fósforo de 1.0 a $13.5 \mathrm{mg} \mathrm{kg}^{-1}$ y la de potasio de 1.18 a 2.37 Cmol (+) $\mathrm{kg}^{-1}$ (Álvarez-Solís et al., 2000), lo anterior concuerda con los valores encontrados en el tepetate de la Hacienda Tepetzala, después del establecimiento de las diferentes poblaciones de L. campestris. Respecto al contenido de materia orgánica, reportaron que en tepetates roturados y con una asociación maíz-haba más una aplicación de 20 t ha ${ }^{-1}$ año $^{-1}$ de estiércol se obtuvo $1.58 \%$ de materia orgánica y un $\mathrm{pH}$ de 7.3 , lo que indica que las prácticas de manejo mejoran de forma sustancial las condiciones de las propiedades edáficas (Prat et al., 2003). Se observó enmuestras de tepetate provenientes del Estado de México incrementos en los valores de algunas propiedades edáficas, como la capacidad de intercambio catiónico de 28.1 Cmol (+) kg-1(Rodríguez-Tapia et al., 2004), la cual es relativamente mayor a la del tepetate de la rizósfera de L. campestris. Por la parte nutrimental, se encontró que la concentración de $\mathrm{N}$ total, así como de fósforo, potasio, calcio y magnesio del tepetate con $L$. campestris no tuvieron un aumento significativo al comparar el valor con el del tepetate 2017, previo a establecer la planta. Sin embargo, es una leguminosa que tiene la capacidad de solubilizar fósforo, debido a su sistema radical y a su potencial de supervivencia en suelos degradados (Vielma, 1999). En este sentido, Gama-Castro et al. (2007) reportan una concentración de $\mathrm{N}$ total de $0.03 \%$ en tepetate, lo que coincide con lo encontrado en los tepetates de la rizósfera de las diferentes poblaciones de L. campestris. Por otro lado, al establecer en tepetate Cassia tomentosa, una leguminosa que se emplea como forraje para alimentación animal se reporta en el sustrato una concentración de $0.001 \%$ de $\mathrm{N}$ total, 0.80 $\mathrm{mg} \mathrm{kg}^{-1}$ de fósforo y $249.1 \mathrm{mg} \mathrm{kg}^{-1}$ de potasio (García-Gallegos et al., 2009), valores inferiores a lo que tuvo el tepetate de la zona rizosférica de L. campestris; a excepción de la concentración de potasio, lo que sugiere que esta leguminosa puede influir en la solubilidad de los nutrimentos.

\section{CONCLUSIONES}

El uso de L. campestris como una práctica de rehabilitación propició una mejora en la concentración de nutrimentos, así como de algunas propiedades edáficas del tepetate, con respecto al tepetate previo a establecer la planta, lo que permite una importante retención de agua, además de un incremento en la actividad microbiana; por lo que convierte a esta leguminosa en una excelente candidata para la rehabilitación de zonas donde aflore el tepetate. En la Hacienda San Antonio Tepetzala, es importante continuar y complementar la 
rehabilitación de los tepetates con otras prácticas sustentables que sean promisorias para mejorar la condición del sustrato, como lo es la incorporación de enmiendas orgánicas, abonos verdes, entre otras.

\section{AGRADECIMIENTOS}

Se hace un amplio reconocimiento a la Sra. María Carmen Rivera S. dueña de la Hacienda San Antonio Tepetzala por permitir esta investigación en su propiedad y al CONACyT por su apoyo para realizar los estudios de posgrado de Cristina Munive Martínez No. Becario 606541.

\section{LITERATURA CITADA}

Álvarez-Solís, J. D., Ferrera, C. R. y Etchevers, B. J. 2000. Actividad microbiana en tepetate con incorporación de residuos orgánicos. Agrociencia 34: 523-532.

Álvarez-Sánchez, M. E. y Marín-Campos, A. 2011. Manual de procedimientos analíticos de suelo y planta. Laboratorio de Química. Departamento de Suelos. Universidad Autónoma Chapingo, Chapingo, Méx. 62 p.

Báez, A., Etchevers, J. D., Prat, C. Márquez, A. y Ascencio, E. 2008. Manejo agronómico de suelos endurecidos (tepetates) del eje Neovolcánico de México. Red Iberoamericana de Física y Química Ambiental. Memoria Red POCAIBA. pp. 69-84.

Callo, C. D. 2013. Prácticas agroforestales y especies de uso múltiple para la rehabilitación y conservación de suelos volcánicos en Adolfo López Mateos, Tlaxcala: percepción de los agricultores. Sociedades Rurales, Producción y Medio Ambiente 13 (4): 117-134.

Dias, L. E., Franco, A. A., Campello, E., De Faria, S. M. y Da Silva, E. M. 1995.
Leguminosas forestales: aspectos relacionados con su nutrición y uso en la recuperación de suelos degradados. Bosque 16(1): 121-127.

https://doi.org/10.4206/bosque.1995.v16 n1-15

DOF. 2002. Diario Oficial de la Federación NOM-021-SEMARNAT-2000 Norma Oficial Mexicana que establece las especificaciones de fertilidad, salinidad y clasificación de suelos, estudio, muestreo y análisis. $89 \mathrm{p}$.

Flores, S. D.; Pérez, M. A. y Navarro. H. 2004. Rehabilitación agroecológica de suelos volcánicos endurecidos, experiencias en el Valle de México. LEISA Revista de Agroecología 1: 24-27.

Fuentes, R. 1971. Comportamiento de la CIC en algunos suelos ácidos de origen volcánico. Tesis M.S.C. Turrialba, Costa Rica, IICA, 107 p.

Gama-Castro, J., Solleiro-Rebolledo, E., FloresRomán, D., Sedov, S., Cabadas-Báez, H. y Díaz-Ortega, J. 2007. Los tepetates y su dinámica sobre la degradación y el riesgo ambiental: el caso del Glacis de Buenavista, Morelos. Boletín de la Sociedad Geológica Mexicana. LIX. 1: 133-145.

https://doi.org/10.18268/BSGM2007v59 n1a11

García-Gallegos, E., Gómez-Cruz, G., Vázquez-Cuecuecha, O. G. y ZamoraCampos, E.M. 2009. Respuesta de Cassia tomentosa desarrollada en tepetate con inoculación micorrízica. Revista UDO Agrícola 9 (4): 816-825.

García, C., Flores, D., García, N. E. y Ferrera, R. 2008. Efecto de enmiendas orgánicas, higuera y micorrizasobre las características de un tepetate. Terra Latinoamericana. 26: 309-315. 
Gutiérrez, N. P., De León, G. F., Etchevers, B. J. y Casas, F. A. 2010. Effect of scarification, self-inhibition, and sowing depth on seed germination of Lupinus campestris. Chilean Journal of Agricultural Research 70 (3): 365-371. https://doi.org/10.4067/S0718-58392010 000300003

INEGI. 2009. Instituto Nacional de Estadística Geografía e Informática - Prontuario de información geográfica municipal de los Estados Unidos Mexicanos. Atlangatepec, Tlaxcala. Clave geoestadística 29003.

Pajares-Moreno, S., Gallardo-Lancho, J. F., Marinari, S. y Etchevers-Barra, J. D. 2010. Indicadores bioquímicos de calidad en tepetates cultivados del eje Neovolcánico Mexicano. Agrociencia 44 (2): 261-274.

Prat, Ch., Ordaz, Ch. V. y Rugama, U. J. A. 2003. Impacto de la roturación y el manejo agronómico de un tepetate sobre su estructura. Terra 21:109-115.

Rodríguez-Tapia, S., Ortiz-Solorio, C. A., Hidalgo-Moreno, C. y GutiérrezCastorena, M. del C. 2004. Los tepetates de la ladera oeste del cerro Tláloc: saprolita, sin endurecimiento pedológico. Terra Latinoamericana 22 (1): 11-21.

Vance, C. P. 2001. Symbiotic nitrogen fixation and phosphorus acquisition. Plant nutrition ina world of declining renewable resources. Plant Physiology 127: 390-397.

https://doi.org/10.1104/pp.127.2.390
Vázquez, A. A. 1997. Guía para interpretar el análisis químico del agua y suelo. Departamento de Suelos. Universidad Autónoma Chapingo. $2^{\mathrm{a}}$. Edición. Chapingo, México. 31 p.

Vázquez, C. O. G. 2017.Variabilidad morfológica y genética de especies del género Lupinus en el estado de Puebla. Tesis de doctorado. Colegio de Postgraduados, Campus Montecillo, estado de México. 149 p.

Velázquez, R. A. S., Flores, D. y Acevedo S. O. A. 2001. Formación de agregados en tepetate porinfluencia de especies vegetales. Agrociencia 35 (3): 311-320.

Vielma, A. M. 1999. Caracterización de cepas autóctonas de Bradyrhizobium spp. Aisladas de Lupinus spp. Revista de la Facultad de Agronóma (LUZ) 16: 495508. 
Copyright (c) 2018 C. Munive Martinez, Oscar Vázquez Cuecuecha, Eurise Zamora Campos, Aline López López, Claudia Irene Calvario Rivera y Elizabeth Garcia G allegos

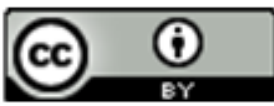

E ste tex to está protegido por una licencia CreativeCommons 4.0.

Usted es libre para Compartir —copiar y redistribuir el $\mathrm{m}$ aterial en cualquier medio o formato-y Adaptar el documento - remezclar, transformar y crear a partir del material- para cualquier propósito, incluso para fines com erciales, siempre que cumpla la condición de:

Atribución: Usted debe dar crédito a la obra original de manera adecuada, proporcionar un enlace a la licencia, e in dicar si se han realizado cambios. Puede hacerlo en cualquier forma razonable, pero no de forma tal que sugiera que tiene el apoyo del licenciante olo recibe por el uso que hace de la obra.

Resumenclelicencia - Textocompletodelalicencia 